OPEN ACCESS

Edited by:

Yumiko Motoi,

Juntendo University, Japan

Reviewed by:

Tadanori Hamano,

University of Fukui, Japan Roland Brandt,

Osnabrück University, Germany

${ }^{*}$ Correspondence: Shuko Takeda takeda@cgt.med.osaka-u.ac.jp

Specialty section:

This article was submitted to Neurodegeneration,

a section of the journal

Frontiers in Neuroscience

Received: 04 September 2019 Accepted: 11 November 2019 Published: 13 December 2019

Citation:

Takeda S (2019) Tau Propagation as a Diagnostic and Therapeutic Target for Dementia: Potentials and Unanswered Questions.

Front. Neurosci. 13:1274. doi: 10.3389/fnins.2019.01274

\section{Tau Propagation as a Diagnostic and Therapeutic Target for Dementia: Potentials and Unanswered Questions}

\author{
Shuko Takeda* \\ Department of Clinical Gene Therapy, Graduate School of Medicine, Osaka University, Suita, Japan
}

A unique clinical course of Alzheimer's disease (AD), beginning with memory deficit as the earliest symptom, is well-correlated with a progressive pattern of intracellular aggregates of tau (neurofibrillary tangles), which spread from the medial temporal lobe to other brain areas in a stereotypical manner. Recent findings from basic research using in vitro and in vivo models demonstrated that pathological forms of extracellular tau can be taken up by cells and induce intracellular tau aggregates. On the basis of these neuropathological observations and experimental findings, the "tau propagation hypothesis" has been proposed, in which the stereotypical spreading of the tau pathology observed in the brain of AD patients can be explained by the interneuron transfer of the pathological form of tau. The concept of tau propagation remains controversial, and many unsolved questions exist; however, it has been attracting attention as a potential therapeutic target for halting AD progression. This article reviews the recent findings regarding the tau propagation hypothesis, including the basic concept and evidence of interneuron tau transfer, potentials as a diagnostic and therapeutic target, and unsolved questions for a better understanding of tau propagation.

Keywords: tau, propagation, dementia, Alzheimer's disease, treatment, diagnosis

\section{INTRODUCTION}

Tau, a microtubule-associated protein mainly expressed in neurons, is involved in polymerizing microtubules and maintaining microtubule stability under physiological conditions (GrundkeIqbal et al., 1986; Lewis and Dickson, 2016; Wang and Mandelkow, 2016). The majority of soluble tau is bound to microtubules, stabilizing them by shifting the equilibrium from free tubulin and microtubules toward polymerization. The physiological functions of tau are highly regulated by a wide range of posttranslational modifications, including phosphorylation, acetylation, glycation, isomerization, nitration, SUMOylation, and ubiquitination (Morris et al., 2011). The alteration of these modifications can affect tau functions and potentially lead to pathological conditions. Hyperphosphorylation of tau causes its detachment from microtubules, thereby impairing the axonal stability and trafficking necessary for normal neuronal activities (Morris et al., 2011). 
Research indicates that the interaction of tau with microtubules is not as stable as previously believed (Janning et al., 2014), and tau exerts diverse functions that interact with multiple binding partners (Morris et al., 2011). Using single-molecule tracking experiments, Janning et al. (2014) showed that tau interacts with microtubules in a highly dynamic manner, with dwell time in the milliseconds range. The dynamic behavior of tau may underlie the regulation of microtubule dynamics and other functions coordinated with multiple binding partners. Tau interacts with plasma membrane-binding protein annexins, which may contribute to the enrichment and distribution of tau intracellularly and contribute to tau's release from the cells (Gauthier-Kemper et al., 2018). The rapid, dynamic behavior of tau may be essential for its non-microtubule-related functions and its interactions with multiple binding partners in the cell (Morris et al., 2011).

Tau is also known as a major component of neurofibrillary tangles (NFTs), one of the cardinal pathological features in Alzheimer's disease (AD) (Hyman, 1997; Serrano-Pozo et al., 2011). A cross-sectional neuropathological study showed that the tau pathology of $\mathrm{AD}$ typically spreads from one area to another in a stereotypical pattern along a neural network (Braak and Braak, 1991). In early stage AD, NFTs appear in the transentorhinal cortex in the medial temporal lobe and later spread across the entire cerebral cortex via the hippocampal areas (Braak staging of NFT) (Braak and Braak, 1991). The mechanism underlying the stereotypical progression pattern of NFT in $\mathrm{AD}$ has not been elucidated; however, findings from recent studies suggest that tau pathology potentially spreads by the interneuronal transfer of the pathological from of tau (Figure 1; Hyman, 2014; Mudher et al., 2017; Gibbons et al., 2019). This phenomenon, called "tau propagation," gains attention as a pathological hypothesis explaining the reason why $\mathrm{AD}$ progresses over time and also as a new therapeutic target for AD.

\section{TAU PATHOLOGY IN THE BRAIN OF PATIENTS WITH DEMENTIA}

\section{Tauopathy and Isoforms of Tau}

A class of neurodegenerative disorders characterized by intracellular aggregates of tau in the brain, including AD, Pick's disease, corticobasal degeneration, progressive supranuclear palsy, and argyrophilic grain disease, are collectively called tauopathy. Six isoforms of tau are known to be present in the human adult brain, and, in terms of the number of microtubule-binding repeats, tau isoforms are divided into two groups: 3-repeat and 4-repeat tau. A distinct pattern of tau accumulation is observed in each tauopathy: tau aggregates are present in either or both forms of tau isoforms - for example, 3-repeat tau in Pick's disease; 4-repeat tau in corticobasal degeneration, progressive supranuclear palsy, and argyrophilic grain disease; and both 3-repeat and 4-repeat tau in $\mathrm{AD}$ (Lewis and Dickson, 2016). The differences in biochemical characteristics, including isoforms, of accumulated tau, are closely related to the pattern of progression of the tau pathology

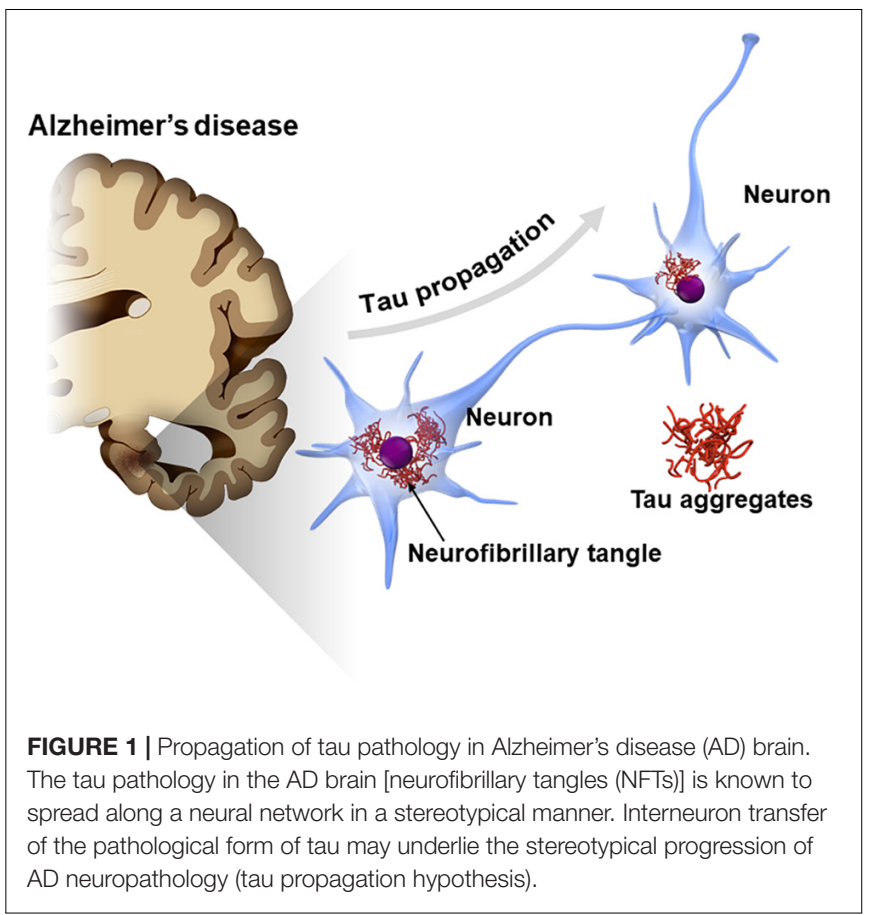

in each tauopathy (initial region and/or patterns of subsequent progression), which is important in understanding the tau propagation hypothesis.

\section{Progression Pattern of Tau Pathology in the Brain of AD Patients}

Neurofibrillary tangles in $\mathrm{AD}$ patients appear first in the transentorhinal cortex or the entorhinal cortex in the medial temporal lobe (Braak stages I and II), then gradually progress to the hippocampal region (Braak stages III and IV), and finally involve the association neocortex or the primary areas of the neocortex (Braak stages V and VI) (Serrano-Pozo et al., 2011). This pattern of NFT progression closely resembles the clinical course of $\mathrm{AD}$, which starts with severe memory deficit and slowly progresses to another cognitive dysfunction, indicating that the spread of the tau pathology is deeply associated with neurological dysfunction (Qian et al., 2017; DeVos et al., 2018). Since the progression of the tau pathology appears to spread along neuroanatomical connections, in other words, a brain region to another via axonal projections, the tau propagation hypothesis has been proposed, in which the pathological form of tau transfers between neurons (Clavaguera et al., 2013; Walker et al., 2013).

\section{TAU PROPAGATION}

\section{Tau Propagation in vitro}

In vitro experiments performed by Frost et al. (2009) showed that a tau seed added to a culture medium can be taken up into cells via endocytosis and form new intracellular aggregates of tau. This finding provided theoretical evidence for the interneuronal transfer of tau as a mechanism underlying tau propagation. 
TABLE 1 | The nature of tau species involved in propagation.

\begin{tabular}{|c|c|c|c|}
\hline The nature of tau species & Origin of tau seeds & Experimental model & References \\
\hline Tau monomer & Recombinant tau & Tau uptake in cell culture model & Michel et al. (2014) \\
\hline Tau trimers & $\begin{array}{l}\text { Recombinant tauBrain-derived } \\
\text { tau from AD brain }\end{array}$ & Tau uptake in cell culture model & Mirbaha et al. (2015) \\
\hline Tau oligomers (dimer/trimer) & Brain-derived tau from AD brain & $\begin{array}{l}\text { Intracereberal injections of tau seeds in } \\
\text { mouse model }\end{array}$ & Lasagna-Reeves et al. (2012) \\
\hline $\begin{array}{l}\text { Low-molecular-weight aggregates (spherical } \\
\text { oligomers with diameters ranging from } 10 \text { to } 30 \mathrm{~nm} \text { ) }\end{array}$ & Recombinant tau & $\begin{array}{l}\text { Trans-synaptic transfer of tau in } \\
\text { microfluidic devices }\end{array}$ & Wu et al. (2013) \\
\hline Phosphorylated large tau oligomers (>10 mers) & $\begin{array}{l}\text { Brain-derived tau from } \\
\text { tau-transgenic mice }\end{array}$ & $\begin{array}{l}\text { Tau uptake in cell culture } \\
\text { modellntracereberal injections of tau } \\
\text { seeds in mouse model }\end{array}$ & Jackson et al. (2016) \\
\hline $\begin{array}{l}\text { Phosphorylated high-molecular-weight tau } \\
\text { (>600 kDa) }\end{array}$ & $\begin{array}{l}\text { Brain-derived tau from AD } \\
\text { brainBrain-derived tau from } \\
\text { tau-transgenic mice }\end{array}$ & $\begin{array}{l}\text { Tau uptake in cell culture } \\
\text { modelTrans-synaptic transfer of tau in } \\
\text { microfluidic devicesIntracereberal } \\
\text { injections of tau seeds in mouse model }\end{array}$ & Takeda et al. (2015) \\
\hline Tau aggregates & Recombinant tau & Tau uptake in cell culture model & Frost et al. (2009) \\
\hline Tau aggregates & $\begin{array}{l}\text { Exosomal tau from primary } \\
\text { neurons or cerebrospinal fluid } \\
\text { of } A D \text { patients }\end{array}$ & $\begin{array}{l}\text { Tau uptake in cell culture } \\
\text { modelTrans-synaptic transfer of tau in } \\
\text { microfluidic devices }\end{array}$ & Wang et al. (2017) \\
\hline Tau fibrils & Recombinant tau & $\begin{array}{l}\text { Intracereberal injections of tau seeds in } \\
\text { mouse model }\end{array}$ & lba et al. (2013) \\
\hline Tau fibrils & Brain-derived tau from AD brain & $\begin{array}{l}\text { Intracereberal injections of tau seeds in } \\
\text { mouse model }\end{array}$ & Guo et al. (2016) \\
\hline
\end{tabular}

$A D$, Alzheimer's disease.

Following the paper by Frost et al. (2009) multiple research groups reported on the mechanisms of the cellular uptake of extracellular tau and subsequent intracellular aggregation. A better understanding of the biochemical features of the tau involved in propagation is important in developing therapeutic strategies (Sanders et al., 2014; Panza et al., 2016). The first report by Frost et al. (2009) showed that the fibrillar form of tau is more easily taken up into the cells than the monomeric form of tau. On the other hand, Mirbaha et al. (2015) reported that the tau trimer is the minimal unit that is capable of inducing intracellular aggregates of tau. Wu et al. (2013) and Takeda et al. (2015) performed experiments using a unique chamber for neuronal cell culture with a microfluidic chip demonstrating that the tau oligomer is more easily propagated than the tau monomer. Another report demonstrated that the monomeric form of tau can mediate tau propagation (Michel et al., 2014). To date, which form of tau is really involved in interneuronal propagation remains controversial (Table 1).

The aggregation and propagation properties of tau also depend on its isoforms (Nonaka et al., 2010; Dinkel et al., 2011). Variations of the tau isoform can affect posttranslational modification, such as the fragmentation and phosphorylation of tau, which can potentially modify intracellular uptake and aggregation capability (Despres et al., 2017). The propagation activity of tau can also be affected by the type of genetic mutation or the origin of tau (synthetic or brain-derived tau protein) (Lewis and Dickson, 2016).

\section{Tau Propagation in Animal Models}

Clavaguera et al. (2009) reported that intracellular tau aggregates can be induced in the mouse brain that was injected with seed-competent tau aggregates, demonstrating that tau propagation may occur even in vivo. In this experiment, brain homogenates from tau-transgenic mice that overexpress the mutant form of tau in the brain were injected into another mouse brain that does not have the tau pathology; 12 months later, tau aggregates were observed in the neurons of the recipient mouse brain.

In 2012, three research groups independently reported almost similar mouse models of tau propagation (de Calignon et al., 2012; Harris et al., 2012; Liu et al., 2012). Transgenic mice overexpressing human mutant tau (P301L) only in the entorhinal cortex were generated using a region-specific promoter, and the neuropathological changes were examined over time up to 2 years. First, the tau pathology appeared in the entorhinal cortex (where the human mutant tau was overexpressed) at the age of 12 months and then appeared in the neurons of the dentate gyrus, which has strong neuroanatomical connection to the entorhinal cortex but is not supposed to express the human mutant tau, at the age of about 18 months. These experiments demonstrated that, although there are some problems/limitations including the region specificity of the promoter, tau pathologies that comprise the human mutant tau in neurons of the entorhinal cortex can potentially spread likely via synaptic transmission to neurons in the dentate gyrus, a major target of axonal projections.

Along the same line, Iba et al. (2013) and Ahmed et al. (2014) conducted in vivo experiments wherein the fibrillar form of recombinant tau, or tau-transgenic mouse brainderived tau, was injected into a specific region of the mouse brain that does harbor the tau pathology. Newly induced tau pathology appeared in the brain regions along a neural network 
from the injection site. The region where the tau pathology appeared was not related to the distance from the tau seed injection site but to a neuroanatomical connection (along the axonal projection), implying that tau propagation possibly occurs via synapses.

\section{Mechanism of Tau Propagation}

Although researchers have demonstrated that certain forms of pathological tau have the property of transmission between neurons (Calafate et al., 2015), the molecular mechanisms underlying tau propagation are still largely unknown. The interneuronal propagation of tau is divided into three steps: the intracellular pathological tau (1) is released into the extracellular space, (2) is taken up by recipient cells, and (3) forms new intracellular aggregates in the recipient cells (Figure 2).

Regarding tau release into the extracellular space, passive leakage from degenerated cells and tau dissociation from ghost tangles likely contribute to it. Recent studies have shown a possibility that physiological active tau release could occur without neurodegeneration (Yamada et al., 2011, 2014; Pooler et al., 2013), which may be involved in tau propagation (Wu et al., 2016). The cellular uptake of extracellular tau can potentially be mediated by endocytosis (Holmes et al., 2013) occurring on the cell surface or during synaptic transmission. A recent study by Falcon et al. (2018) showed that seed-competent tau enters cells via clathrin-independent endocytosis and escape from damaged endomembranes into the cytosol, triggering cytosolic tau aggregation. The galectin8-dependent autophagy system mediated the delivery of tau seeds from the endo-lysosomal pathway to the cytosol, implying a role for autophagy in intracellular tau aggregation and propagation.

Tau propagation is known to occur in both directions (retrograde and anterograde) along a neural network (Ahmed et al., 2014; Takeda et al., 2015), which indicates that tau propagation does not necessarily occur only via synaptic transmission. Asai et al. and other researchers reported that exosomes are also involved in the mechanism that mediates interneuronal tau transfer (Asai et al., 2015; Wang et al., 2017).

\section{Propagation of Other Pathological Proteins}

Researchers have reported on other proteins implicated in neurodegenerative diseases, including amyloid- $\beta$ (Petkova et al., 2005; Watts et al., 2014; Sengupta et al., 2016; Condello and Stoehr, 2018; Ruiz-Riquelme et al., 2018) or $\alpha$-synuclein (Dehay et al., 2016; McCann et al., 2016; Steiner et al., 2018), which show the key biochemical properties and capabilities of cell-tocell propagation. Although they show distinct distributions in the human brain, misfolded forms of these pathological proteins may propagate through mechanisms similar to tau (Goedert et al., 2017). In the brains of AD patients, amyloid- $\beta$ plaques develop in the basal temporal and orbitofrontal cortex in the early phase before reaching other areas, such as the neocortex, hippocampal formation, and basal ganglia, and then finally spreading to the lower brain stem. Inclusions of $\alpha$-synuclein first develop in the peripheral nervous system and olfactory bulb, ascend to the brainstem and midbrain, and then spread to the basal forebrain and neocortex (Braak et al., 2003; Goedert et al., 2017). Distinct conformations of each pathological protein may

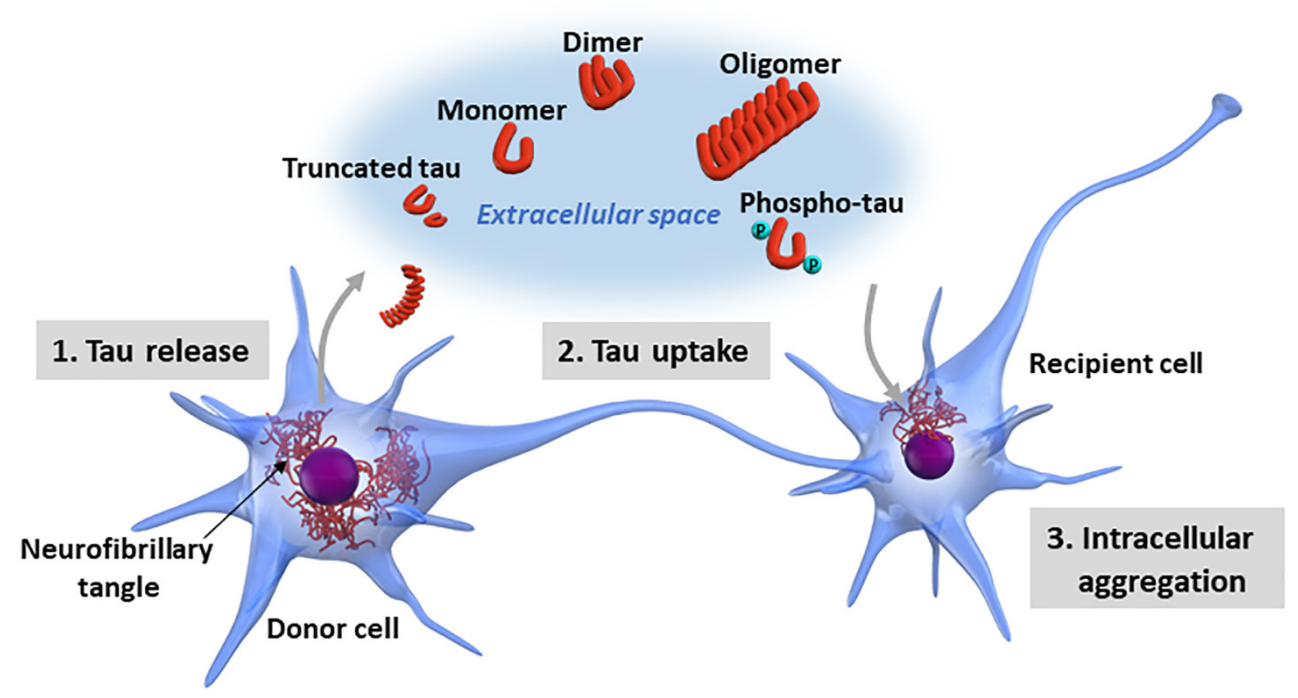

FIGURE 2 | Neuron-to-neuron transfer of tau. The processes involved in tau propagation can be divided into three basic steps: (1) the pathological form of tau is released into the extracellular space from the donor cell; (2) the pathological tau released into the extracellular space is taken up by recipient cells; and (3) the pathological tau taken up into the recipient cells forms new intracellular aggregates. Tau exists in various forms in terms of biochemical property, including monomeric, oligomeric, truncated, and phosphorylated forms. Additionally, tau may undergo a wide range of posttranslational modifications, including acetylation, glycation, isomerization, nitration, SUMOylation, and ubiquitination or a mixture of these modifications. It is still largely unknown which forms of tau are released into the extracellular space and involved in tau propagation. 
determine the distribution pattern and speed of spread and may underlie the phenotypic diversity of neurodegenerative diseases. Although the molecular mechanisms mediating the propagation of pathological proteins have not been fully understood, they can be specific targets for therapeutic interventions.

\section{UNSOLVED QUESTIONS REGARDING TAU PROPAGATION HYPOTHESIS}

Tau propagation is an intriguing pathological hypothesis for explaining the fundamental characteristics of dementia that progresses over time, but many questions regarding its mechanisms or the validity of the hypothesis still remain unsolved.

It is necessary to carefully examine how exactly the tau pathology in cultured cells and brains of animal models reflects the tau pathology observed in the human brain. So far, there is no clear evidence showing biochemical and biological similarities between the structures of tau aggregates in cultured cells or mouse brains and those of the NFTs in AD patients.

In humans, the accumulation of tau aggregates is accompanied by various cognitive dysfunctions associated with neuronal death; however, with some exceptions, the tau pathology induced in cell lines or mouse brains is often not accompanied by neuronal death or dysfunction. This may be because the tau seeds used in experimental models for propagation study are not identical to the species that exert neurotoxicity in human tauopathies. Moreover, scientists do not know which species of tau is really involved in neurotoxicity (Ballatore et al., 2007). Propagation property and neurotoxicity may be independent features of distinct forms of tau. Additionally, most experiments based on cell or animal models with overexpression of the mutant form of tau may not accurately reflect the brain pathology in patients with sporadic AD without genetic mutation.

There are some issues regarding the validity of the concept of tau propagation. In the tau propagation hypothesis, the medial temporal lobe is considered an initial site of origin for tau propagation starting in the brain of $\mathrm{AD}$ patients. However, the tau pathology in $\mathrm{AD}$ is known to begin in areas such as the dorsal raphe nuclei (Grinberg et al., 2009) and the locus coeruleus (Satoh and Iijima, 2019) or it can also begin in the area of the neocortex rather than the medial temporal lobe (Braak and Braak, 1991; Lewis and Dickson, 2016). The dorsal raphe nuclei and locus coeruleus are connected with the transentorhinal cortex where tau pathology develops in the early stages of $\mathrm{AD}$. These nuclei may be affected by tau pathology even before the transentorhinal cortex, and they may act as an initiation site for the subsequent spread of tau pathology throughout the brain (Grinberg et al., 2009; Satoh and Iijima, 2019).

The heterogenicity of the distribution of the AD tau pathology should also be taken into consideration when understanding the propagation hypothesis; the initial site of origin for tau propagation may not be limited to only one site, but multiple brain regions could be the starting points of propagation instead. Tau aggregates also appear in glial cells in some types of tauopathies, but the applicability of the tau propagation hypothesis to glial tau pathology remains unclear.

Some fundamental issues have not been solved, including why conformational changes and aggregation initiate at the initial site of origin for tau propagation. What is the most upstream trigger of tau aggregation in the initial site? In human tauopathy, even in cases caused by genetic mutation in which all cells are supposed to uniformly possess the same mutation, a striking laterality of neuropathology is occasionally observed. Can the tau propagation hypothesis explain the asymmetricity observed in some cases of tauopathy? These unsolved questions need to be discussed and answered for a better understanding of tau propagation.

\section{FUTURE DIRECTIONS: TOWARD DIAGNOSIS AND TREATMENT BASED ON THE TAU PROPAGATION HYPOTHESIS}

A stereotypical pattern of the progression of the tau pathology in the brains of patients with sporadic AD may be explained by the tau propagation hypothesis, which can potentially lead to the development of diagnostic and treatment strategies for $\mathrm{AD}$ (Sigurdsson, 2016; Takeda, 2019).

Takeda et al. (2016) examined the biological and biochemical properties of brain extracellular tau from various sources including lumbar cerebrospinal fluid (CSF) from AD patients. They found that the bioactive tau species involved in propagation is present in the CSF from $\mathrm{AD}$ patients, and its concentrations were significantly higher than those in control subjects or patients with frontotemporal dementia. This finding suggests that the tau species involved in propagation could be useful as a biomarker for $\mathrm{AD}$, specifically for monitoring tau propagation activity.

The tau propagation hypothesis, based on the concept that the clinical progression of $\mathrm{AD}$ is linked with the spreading of the tau pathology, supports the idea that clearing the tau involved in propagation may slow the spread of the tau pathology and possibly of cognitive decline (Nicholls et al., 2017; Nobuhara et al., 2017). The potential efficacy of antibodybased therapeutics targeting tau has been demonstrated in several studies using animal models (Yanamandra et al., 2013; Pedersen and Sigurdsson, 2015), although it is unclear how an antibody can exert its therapeutic efficacy against an intracellular protein such as tau. In this regard, the tau propagation hypothesis may provide a rationale for the antibody-based strategy targeting tau propagation that could be mediated by extracellular tau, which can be captured and neutralized by an anti-tau antibody; the antibody does not need to be incorporated into the cell to exert its therapeutic efficacy (Takeda, 2019).

Identifying the most efficient epitope should be critical in therapeutic development for tau-based immunotherapy. So far, various antibodies against distinct epitopes of tau have been tested (Takeda, 2019). Some antibodies successfully ameliorated the cognitive deficit and neuropathology in mouse models. Nobuhara et al. (2017) used a cell culture model to assess the 
effect of multiple anti-tau antibodies on tau propagation to identify suitable target epitopes for blocking tau uptake and propagation. The blocking efficacy varied depending on the epitope that each anti-tau antibody targeted, and the antibody against the N-terminal and phospho-site of tau showed the most effectiveness (Nobuhara et al., 2017). Other processes involved in tau propagation, including tau release, cellular uptake, and intracellular aggregation, could be therapeutic targets for halting the spread of the tau pathology, which need further investigation.

\section{CONCLUSION}

This article reviewed the recent findings regarding the tau propagation hypothesis, namely, the basic concept and evidence of tau propagation, potential as a diagnostic and therapeutic target, and unsolved questions. The hypothesis has been attracting considerable attention in the research field of ADbecause it may explain the stereotypical progression of the tau

\section{REFERENCES}

Ahmed, Z., Cooper, J., Murray, T. K., Garn, K., McNaughton, E., Clarke, H., et al. (2014). A novel in vivo model of tau propagation with rapid and progressive neurofibrillary tangle pathology: the pattern of spread is determined by connectivity, not proximity. Acta Neuropathol. 127, 667-683. doi: 10.1007/ s00401-014-1254-6

Asai, H., Ikezu, S., Tsunoda, S., Medalla, M., Luebke, J., Haydar, T., et al. (2015). Depletion of microglia and inhibition of exosome synthesis halt tau propagation. Nat. Neurosci. 18, 1584-1593. doi: 10.1038/nn. 4132

Ballatore, C., Lee, V. M., and Trojanowski, J. Q. (2007). Tau-mediated neurodegeneration in Alzheimer's disease and related disorders. Nat. Rev. Neurosci. 8, 663-672. doi: 10.1038/nrn2194

Braak, H., and Braak, E. (1991). Neuropathological stageing of Alzheimerrelated changes. Acta Neuropathol. 82, 239-259. doi: 10.1007/bf0030 8809

Braak, H., Rub, U., Gai, W. P., and Del Tredici, K. (2003). Idiopathic Parkinson's disease: possible routes by which vulnerable neuronal types may be subject to neuroinvasion by an unknown pathogen. J. Neural Transm. 110, 517-536. doi: 10.1007/s00702-002-0808-2

Calafate, S., Buist, A., Miskiewicz, K., Vijayan, V., Daneels, G., de Strooper, B., et al. (2015). Synaptic contacts enhance cell-to-cell Tau pathology propagation. Cell Rep. 11, 1176-1183. doi: 10.1016/j.celrep.2015. 04.043

Clavaguera, F., Bolmont, T., Crowther, R. A., Abramowski, D., Frank, S., Probst, A., et al. (2009). Transmission and spreading of tauopathy in transgenic mouse brain. Nat. Cell Biol. 11, 909-913. doi: 10.1038/ncb 1901

Clavaguera, F., Lavenir, I., Falcon, B., Frank, S., Goedert, M., and Tolnay, M. (2013). "Prion-like" templated misfolding in tauopathies. Brain Pathol. 23, 342-349. doi: 10.1111/bpa.12044

Condello, C., and Stoehr, J. (2018). Abeta propagation and strains: implications for the phenotypic diversity in Alzheimer's disease. Neurobiol. Dis. 109, 191-200. doi: 10.1016/j.nbd.2017.03.014

de Calignon, A., Polydoro, M., Suarez-Calvet, M., William, C., Adamowicz, D. H., Kopeikina, K. J., et al. (2012). Propagation of tau pathology in a model of early Alzheimer's disease. Neuron 73, 685-697. doi: 10.1016/j.neuron.2011. 11.033

Dehay, B., Vila, M., Bezard, E., Brundin, P., and Kordower, J. H. (2016). Alphasynuclein propagation: new insights from animal models. Mov. Disord. 31, 161-168. doi: $10.1002 / \mathrm{mds} .26370$ pathology in the brain of $\mathrm{AD}$ patients and provides a rationale for tau-based therapies. Numerous questions regarding the detailed molecular mechanisms underlying the tau propagation remain unanswered. Identifying the specific tau species involved in propagation, molecules mediating tau release and uptake, and surrogate markers for propagation activity should be the key research targets for tackling tau propagation.

\section{AUTHOR CONTRIBUTIONS}

The author confirms being the sole contributor of this work and has approved it for publication.

\section{FUNDING}

This work was supported by JSPS KAKENHI Grant Number 17H05080 [Grant-in-Aid for Young Scientists (A)].

Despres, C., Byrne, C., Qi, H., Cantrelle, F. X., Huvent, I., Chambraud, B., et al. (2017). Identification of the Tau phosphorylation pattern that drives its aggregation. Proc. Natl. Acad. Sci. U.S.A. 114, 9080-9085. doi: 10.1073/pnas. 1708448114

DeVos, S. L., Corjuc, B. T., Oakley, D. H., Nobuhara, C. K., Bannon, R. N., Chase, A., et al. (2018). Synaptic Tau seeding precedes Tau pathology in human Alzheimer's disease brain. Front. Neurosci. 12:267. doi: 10.3389/fnins.2018. 00267

Dinkel, P. D., Siddiqua, A., Huynh, H., Shah, M., and Margittai, M. (2011). Variations in filament conformation dictate seeding barrier between three- and four-repeat tau. Biochemistry 50, 4330-4336. doi: 10.1021/bi200 4685

Falcon, B., Noad, J., McMahon, H., Randow, F., and Goedert, M. (2018). Galectin-8-mediated selective autophagy protects against seeded tau aggregation. J. Biol. Chem. 293, 2438-2451. doi: 10.1074/jbc.M117.80 9293

Frost, B., Jacks, R. L., and Diamond, M. I. (2009). Propagation of tau misfolding from the outside to the inside of a cell. J. Biol. Chem. 284, 12845-12852. doi: 10.1074/jbc.M808759200

Gauthier-Kemper, A., Suarez Alonso, M., Sundermann, F., Niewidok, B., Fernandez, M. P., Bakota, L., et al. (2018). Annexins A2 and A6 interact with the extreme $\mathrm{N}$ terminus of tau and thereby contribute to tau's axonal localization. J. Biol. Chem. 293, 8065-8076. doi: 10.1074/jbc.RA117.00 0490

Gibbons, G. S., Lee, V. M. Y., and Trojanowski, J. Q. (2019). Mechanisms of cellto-cell transmission of pathological Tau: a review. JAMA Neurol. 76, 101-108. doi: 10.1001/jamaneurol.2018.2505

Goedert, M., Masuda-Suzukake, M., and Falcon, B. (2017). Like prions: the propagation of aggregated tau and alpha-synuclein in neurodegeneration. Brain 140, 266-278. doi: 10.1093/brain/aww230

Grinberg, L. T., Rub, U., Ferretti, R. E., Nitrini, R., Farfel, J. M., Polichiso, L., et al. (2009). The dorsal raphe nucleus shows phospho-tau neurofibrillary changes before the transentorhinal region in Alzheimer's disease. A precocious onset? Neuropathol. Appl. Neurobiol. 35, 406-416. doi: 10.1111/j.1365-2990. 2009.00997.x

Grundke-Iqbal, I., Iqbal, K., Quinlan, M., Tung, Y. C., Zaidi, M. S., and Wisniewski, H. M. (1986). Microtubule-associated protein tau. A component of Alzheimer paired helical filaments. J. Biol. Chem. 261, 6084-6089.

Guo, J. L., Narasimhan, S., Changolkar, L., He, Z., Stieber, A., Zhang, B., et al. (2016). Unique pathological tau conformers from Alzheimer's brains transmit tau pathology in nontransgenic mice. J. Exp. Med. 213, 2635-2654. doi: 10. 1084/jem.20160833 
Harris, J. A., Koyama, A., Maeda, S., Ho, K., Devidze, N., Dubal, D. B., et al. (2012). Human P301L-mutant tau expression in mouse entorhinalhippocampal network causes tau aggregation and presynaptic pathology but no cognitive deficits. PLoS One 7:e45881. doi: 10.1371/journal.pone.004 5881

Holmes, B. B., DeVos, S. L., Kfoury, N., Li, M., Jacks, R., Yanamandra, K., et al. (2013). Heparan sulfate proteoglycans mediate internalization and propagation of specific proteopathic seeds. Proc. Natl. Acad. Sci. U.S.A. 110, E3138-E3147. doi: $10.1073 /$ pnas. 1301440110

Hyman, B. T. (1997). The neuropathological diagnosis of Alzheimer's disease: clinical-pathological studies. Neurobiol. Aging 18, S27-S32.

Hyman, B. T. (2014). Tau propagation, different tau phenotypes, and prionlike properties of tau. Neuron 82, 1189-1190. doi: 10.1016/j.neuron.2014. 06.004

Iba, M., Guo, J. L., McBride, J. D., Zhang, B., Trojanowski, J. Q., and Lee, V. M. (2013). Synthetic tau fibrils mediate transmission of neurofibrillary tangles in a transgenic mouse model of Alzheimer's-like tauopathy. J. Neurosci. 33, 1024-1037. doi: 10.1523/JNEUROSCI.2642-12. 2013

Jackson, S. J., Kerridge, C., Cooper, J., Cavallini, A., Falcon, B., Cella, C. V., et al. (2016). Short fibrils constitute the major species of seed-competent Tau in the brains of mice transgenic for human P301S Tau. J. Neurosci. 36, 762-772. doi: 10.1523/jneurosci.3542-15.2016

Janning, D., Igaev, M., Sundermann, F., Bruhmann, J., Beutel, O., Heinisch, J. J., et al. (2014). Single-molecule tracking of tau reveals fast kiss-and-hop interaction with microtubules in living neurons. Mol. Biol. Cell 25, 3541-3551. doi: 10.1091/mbc.E14-06-1099

Lasagna-Reeves, C. A., Castillo-Carranza, D. L., Sengupta, U., Guerrero-Munoz, M. J., Kiritoshi, T., Neugebauer, V., et al. (2012). Alzheimer brain-derived tau oligomers propagate pathology from endogenous tau. Sci. Rep. 2:700. doi: 10.1038/srep00700

Lewis, J., and Dickson, D. W. (2016). Propagation of tau pathology: hypotheses, discoveries, and yet unresolved questions from experimental and human brain studies. Acta Neuropathol. 131, 27-48. doi: 10.1007/s00401-015$1507-\mathrm{z}$

Liu, L., Drouet, V., Wu, J. W., Witter, M. P., Small, S. A., Clelland, C., et al. (2012). Trans-synaptic spread of tau pathology in vivo. PLoS One 7:e31302. doi: 10.1371/journal.pone. 0031302

McCann, H., Cartwright, H., and Halliday, G. M. (2016). Neuropathology of alphasynuclein propagation and braak hypothesis. Mov. Disord. 31, 152-160. doi: $10.1002 / \mathrm{mds} .26421$

Michel, C. H., Kumar, S., Pinotsi, D., Tunnacliffe, A., St George-Hyslop, P., Mandelkow, E., et al. (2014). Extracellular monomeric tau protein is sufficient to initiate the spread of tau protein pathology. J. Biol. Chem. 289, 956-967. doi: 10.1074/jbc.M113.515445

Mirbaha, H., Holmes, B. B., Sanders, D. W., Bieschke, J., and Diamond, M. I. (2015). Tau Trimers are the minimal propagation unit spontaneously internalized to seed intracellular aggregation. J. Biol. Chem. 290, 14893-14903. doi: 10.1074/jbc.M115.652693

Morris, M., Maeda, S., Vossel, K., and Mucke, L. (2011). The many faces of tau. Neuron 70, 410-426. doi: 10.1016/j.neuron.2011.04.009

Mudher, A., Colin, M., Dujardin, S., Medina, M., Dewachter, I., Alavi Naini, S. M., et al. (2017). What is the evidence that tau pathology spreads through prionlike propagation? Acta Neuropathol. Commun. 5:99. doi: 10.1186/s40478-0170488-7

Nicholls, S. B., DeVos, S. L., Commins, C., Nobuhara, C., Bennett, R. E., Corjuc, D. L., et al. (2017). Characterization of TauC3 antibody and demonstration of its potential to block tau propagation. PLoS One 12:e0177914. doi: 10.1371/journal. pone.0177914

Nobuhara, C. K., DeVos, S. L., Commins, C., Wegmann, S., Moore, B. D., Roe, A. D., et al. (2017). Tau antibody targeting pathological species blocks neuronal uptake and interneuron propagation of Tau in vitro. Am. J. Pathol. 187, 13991412. doi: 10.1016/j.ajpath.2017.01.022

Nonaka, T., Watanabe, S. T., Iwatsubo, T., and Hasegawa, M. (2010). Seeded aggregation and toxicity of \{alpha\}-synuclein and tau: cellular models of neurodegenerative diseases. J. Biol. Chem. 285, 34885-34898. doi: 10.1074/jbc. M110.148460
Panza, F., Solfrizzi, V., Seripa, D., Imbimbo, B. P., Lozupone, M., Santamato, A., et al. (2016). Tau-Centric targets and drugs in clinical development for the treatment of Alzheimer's disease. Biomed. Res. Int. 2016:3245935. doi: 10.1155/ 2016/3245935

Pedersen, J. T., and Sigurdsson, E. M. (2015). Tau immunotherapy for Alzheimer's disease. Trends Mol. Med. 21, 394-402.

Petkova, A. T., Leapman, R. D., Guo, Z., Yau, W. M., Mattson, M. P., and Tycko, R. (2005). Self-propagating, molecular-level polymorphism in Alzheimer's beta-amyloid fibrils. Science 307, 262-265. doi: 10.1126/science.110 5850

Pooler, A. M., Phillips, E. C., Lau, D. H., Noble, W., and Hanger, D. P. (2013). Physiological release of endogenous tau is stimulated by neuronal activity. EMBO Rep. 14, 389-394. doi: 10.1038/embor. 2013.15

Qian, J., Hyman, B. T., and Betensky, R. A. (2017). Neurofibrillary tangle stage and the rate of progression of Alzheimer symptoms: modeling using an autopsy cohort and application to clinical trial design. JAMA Neurol. 74, 540-548. doi: 10.1001/jamaneurol.2016.5953

Ruiz-Riquelme, A., Lau, H. H. C., Stuart, E., Goczi, A. N., Wang, Z., SchmittUlms, G., et al. (2018). Prion-like propagation of beta-amyloid aggregates in the absence of APP overexpression. Acta Neuropathol. Commun. 6:26. doi: 10.1186/s40478-018-0529-x

Sanders, D. W., Kaufman, S. K., DeVos, S. L., Sharma, A. M., Mirbaha, H., Li, A., et al. (2014). Distinct tau prion strains propagate in cells and mice and define different tauopathies. Neuron 82, 1271-1288. doi: 10.1016/j.neuron.2014. 04.047

Satoh, A., and Iijima, K. M. (2019). Roles of tau pathology in the locus coeruleus (LC) in age-associated pathophysiology and Alzheimer's disease pathogenesis: potential strategies to protect the LC against aging. Brain Res. 1702, 17-28. doi: 10.1016/j.brainres.2017.12.027

Sengupta, U., Nilson, A. N., and Kayed, R. (2016). The role of amyloid-beta oligomers in toxicity, propagation, and immunotherapy. EBioMedicine 6, 4249. doi: 10.1016/j.ebiom.2016.03.035

Serrano-Pozo, A., Frosch, M. P., Masliah, E., and Hyman, B. T. (2011). Neuropathological alterations in Alzheimer disease. Cold Spring Harb. Perspect. Med. 1:a006189. doi: 10.1101/cshperspect.a00 6189

Sigurdsson, E. M. (2016). Tau immunotherapy. Neurodegener. Dis. 16, 34-38. doi: $10.1159 / 000440842$

Steiner, J. A., Quansah, E., and Brundin, P. (2018). The concept of alpha-synuclein as a prion-like protein: ten years after. Cell Tissue Res. 373, 161-173. doi: 10. 1007/s00441-018-2814-1

Takeda, S. (2019). Progression of Alzheimer's disease, tau propagation, and its modifiable risk factors. Neurosci. Res. 141, 36-42. doi: 10.1016/j.neures.2018. 08.005

Takeda, S., Commins, C., DeVos, S. L., Nobuhara, C. K., Wegmann, S., Roe, A. D., et al. (2016). Seed-competent high-molecular-weight tau species accumulates in the cerebrospinal fluid of Alzheimer's disease mouse model and human patients. Ann. Neurol. 80, 355-367. doi: 10.1002/ana. 24716

Takeda, S., Wegmann, S., Cho, H., DeVos, S. L., Commins, C., Roe, A. D., et al. (2015). Neuronal uptake and propagation of a rare phosphorylated high-molecular-weight tau derived from Alzheimer's disease brain. Nat. Commun. 6:8490. doi: 10.1038/ncomms 9490

Walker, L. C., Diamond, M. I., Duff, K. E., and Hyman, B. T. (2013). Mechanisms of protein seeding in neurodegenerative diseases. JAMA Neurol. 70, 304-310.

Wang, Y., Balaji, V., Kaniyappan, S., Kruger, L., Irsen, S., Tepper, K., et al. (2017). The release and trans-synaptic transmission of Tau via exosomes. Mol. Neurodegener. 12:5. doi: 10.1186/s13024-0160143-y

Wang, Y., and Mandelkow, E. (2016). Tau in physiology and pathology. Nat. Rev. Neurosci. 17, 5-21.

Watts, J. C., Condello, C., Stohr, J., Oehler, A., Lee, J., DeArmond, S. J., et al. (2014). Serial propagation of distinct strains of Abeta prions from Alzheimer's disease patients. Proc. Natl. Acad. Sci. U.S.A. 111, 10323-10328. doi: 10.1073/ pnas. 1408900111 
Wu, J. W., Herman, M., Liu, L., Simoes, S., Acker, C. M., Figueroa, H., et al. (2013). Small misfolded Tau species are internalized via bulk endocytosis and anterogradely and retrogradely transported in neurons. J. Biol. Chem. 288, 1856-1870. doi: 10.1074/jbc.M112.394528

Wu, J. W., Hussaini, S. A., Bastille, I. M., Rodriguez, G. A., Mrejeru, A., Rilett, K., et al. (2016). Neuronal activity enhances tau propagation and tau pathology in vivo. Nat. Neurosci. 19, 1085-1092. doi: 10.1038/nn. 4328

Yamada, K., Cirrito, J. R., Stewart, F. R., Jiang, H., Finn, M. B., Holmes, B. B., et al. (2011). In vivo microdialysis reveals age-dependent decrease of brain interstitial fluid tau levels in P301S human tau transgenic mice. J. Neurosci. 31, 13110-13117. doi: 10.1523/JNEUROSCI.2569-11.2011

Yamada, K., Holth, J. K., Liao, F., Stewart, F. R., Mahan, T. E., Jiang, H., et al. (2014). Neuronal activity regulates extracellular tau in vivo. J. Exp. Med. 211, 387-393. doi: $10.1084 /$ jem.20131685
Yanamandra, K., Kfoury, N., Jiang, H., Mahan, T. E., Ma, S., Maloney, S. E., et al. (2013). Anti-tau antibodies that block tau aggregate seeding in vitro markedly decrease pathology and improve cognition in vivo. Neuron 80, 402-414. doi: 10.1016/j.neuron.2013.07.046

Conflict of Interest: The author declares that the research was conducted in the absence of any commercial or financial relationships that could be construed as a potential conflict of interest.

Copyright $\odot 2019$ Takeda. This is an open-access article distributed under the terms of the Creative Commons Attribution License (CC BY). The use, distribution or reproduction in other forums is permitted, provided the original author(s) and the copyright owner(s) are credited and that the original publication in this journal is cited, in accordance with accepted academic practice. No use, distribution or reproduction is permitted which does not comply with these terms. 